\title{
COVID-19: Symphony or Cacophony of Immunology
}

\author{
Parikshit Sen ${ }^{1}$, Neelam Makwana ${ }^{2}$, Binita Goswami ${ }^{3}$
}

\begin{abstract}
Indian Journal of Medical Biochemistry (2020): 10.5005/jp-journals-10054-0156

Coronavirus disease 2019 (COVID-19) is a form of viral pneumonia that primarily affects the lower respiratory tract, namely bronchioles and alveoli. It is highly contagious as it can be spread by asymptomatic infected persons, and has consequently led to a global pandemic which has been declared by the WHO as a Public Health Emergency of International Concern (PHEIC).
\end{abstract}

Coronavirus disease 2019 abbreviated as COVID-19 has emerged out to be the greatest public health problem in the world. Coronavirus disease 2019 is caused by a novel virus belonging to the coronavirus family, which causes respiratory disease. Coronavirus disease 2019 is a highly contagious disease with person-to-person transmission, and now affecting the whole world through community transmission. The disease has spread throughout the world with varied mortality rates in different countries. The disease has higher mortality rates among people with extremes of age and the presence of comorbidities. The present review attempts to provide a snapshot of the various immunological responses against this disease sometimes with disastrous consequences such as the cytokine storm.

Keywords: Adaptive immunity, Acute respiratory distress syndrome, Cytokine storm, Innate immunity, Pandemic.

\section{Etiology and Virology}

The causative agent of the COVID-19 pandemic is the severe acute respiratory syndrome-coronavirus-2 (SARS-CoV-2), which is a novel, i.e., a new type of virus from the $\beta$ genus of the coronavirus family. Severe acute respiratory syndrome-coronavirus- 2 is a batderived coronavirus, which was first transmitted to humans via an intermediate host suspected to be a pangolin. Severe acute respiratory syndrome-coronavirus-2 can readily evolve by mutation and homologous and non-homologous recombination, which explains the ease with which the virus has crossed interspecies barriers and adapted itself to cause human-human transmission.

Severe acute respiratory syndrome-coronavirus- 2 is an enveloped virus with a single-stranded RNA genome, which encodes 10 proteins including:

- Spike glycoprotein (S protein), consisting of two subunits-S1 and S2.

- Envelope protein (E protein).

- Membrane protein (M protein).

- Nucleocapsid protein (N protein).

\section{TRANSMISSION}

Severe acute respiratory syndrome-coronavirus-2 is transmitted primarily by aerosolized droplets containing SARS-CoV-2 expelled into the air by an infected person sneezing or coughing which may enter the respiratory tract of an uninfected person. It is also transmitted to a less extent by fomites.

\section{Signs, Symptoms, and Diagnostic Findings}

- Clinical manifestations including dry cough, rhinorrhea, sore throat, headache, dizziness, fever, dyspnea, fatigue, myalgia, nausea, vomiting, and diarrhea. \begin{tabular}{l}
\hline 1,2Maulana Azad Medical College, New Delhi, India \\
${ }^{3}$ Department of Biochemistry, Maulana Azad Medical College, New \\
Delhi, India
\end{tabular}

Corresponding Author: Binita Goswami, Department of Biochemistry, Maulana Azad Medical College, New Delhi, India, Phone: +91 9810592004, e-mail: binita.dr@gmail.com

How to cite this article: Sen P, Makwana N, Goswami B. COVID-19: Symphony or Cacophony of Immunology. Indian J Med Biochem 2020;24(2):66-75.

Source of support: Nil

Conflict of interest: None

- In severe cases, patients develop acute respiratory distress syndrome (ARDS) characterized by short/rapid breathing, and cyanosis, metabolic acidosis, septic shock, and coagulopathies. Cardiovascular complications including vaso-occlusive crises are seen in some severe cases.

- Reduced total leukocyte count with neutrophilia and lymphocytopenia, the neutrophil-lymphocyte.

- In severe cases, substantially elevated serum levels of proinflammatory cytokines are present, including IL- 6 and IL-1 $\beta$, as well as IL-2, IL-8, IL-17, granulocyte colony-stimulating factor (G-CSF), GM-CSF, IFNY-induced protein-10 (IP-10), monocyte chemoattractant protein-1 (MCP1), macrophage inflammatory protein-1a (MIP1a), and tumor necrosis factor-a (TNF-a), constituting the cytokine storm, ${ }^{1}$ characterized by fever, hypertension, and hypoxia, which is the principal cause of the ARDS. Also, C-reactive protein and d-dimer levels are found to be abnormally elevated.

- Bilateral patchy shadows and ground-glass opacity in the lungs is seen in chest radiographs of most COVID-19 patients. ${ }^{2}$

\section{DiAgnOSIS}

The diagnosis of COVID-19 is made by real-time reverse transcriptasepolymerase chain reaction ( $\mathrm{rRT}$-PCR) analysis of respiratory samples obtained through nasopharyngeal swab, sputum, or saliva sample, to detect the presence of SARS-CoV-2 RNA. A serological examination using COVID-19 IgG ELISA assay to detect antibodies 
produced against SARS-CoV-2 is also conducted to detect SARSCoV-2 infection.

\section{Pathogenesis, Pathophysiology, and IMMUNOLOGY \\ Virus Entry and Replication}

Severe acute respiratory syndrome-coronavirus-2 infection proceeds through human angiotensin-converting enzyme-2 (ACE-2) receptor-mediated endocytosis. High levels of surface ACE-2 expression are seen in surfactant producing alveolar type II pneumocytes, as well as ciliated and goblet cells in the lower respiratory tract. High ACE-2 expression is also seen in the intestinal epithelium, as well as on cardiac cells and vascular endothelia. The spike (S) glycoprotein present on the surface of SARS-CoV-2 is the primary determinant for host tropism and pathogenicity (Figs 1 to 6).

The S glycoprotein has two subunits-S1 and S2. The S1 subunit has the presence of a receptor-binding domain (RBD). The RBD binds to ACE-2, which serves as its host cell target receptor. ${ }^{4}$ The glutamine 394 residue in the RBD region of SARS-CoV-2 interacts with the lysine 31 residue on the human ACE-2 receptor. Interaction between the RBD and ACE-2 receptor triggers the endocytosis of the SARS-CoV-2 virion, exposing it to endosomal proteases in the host cell, and this leads to the cleavage of the S1 subunit. ${ }^{4}$ This exposes the fusion peptide (FP) region of the S2 subunit, which inserts itself into the host membrane. The S2 subunit also consists of two heptad repeat regions, HR1 and
HR2. ${ }^{4}$ Insertion of the FP region into the membrane of the host cell causes the folding of the S2 subunit which brings the HR1 and HR2 regions in close approximation. This leads to membrane fusion and the release of the SARS-CoV-2 RNA genome into the cytoplasm of the host cell. Thus, the host cell gets infected with SARS-CoV-2 virion.

Since the SARS-CoV-2 RNA genome is positive, i.e., sense, it can undergo translation without integration into the host DNA genome via cDNA. The SARS-CoV-2 positive (+) ssRNA genome undergoes translation by host ribosomes to produce RNA-dependent RNA polymerase (Replicase), as well as Helicase (to unwind dsRNA). Non-structural proteins 1-16 (nsp 1-16), which form a part of the replication-transcription complex (RTC), are encoded by the open reading frames $1 a$ and $1 b(O R F 1 a / 1 b)$ of the SARS-CoV-2 genomic RNA. The nsp 1-11 are coded for by the ORF1a, and the nsp 12-16 are coded for by the ORF1b. Initially, the ORF 1a and ORF 1b are translated to form two polyproteins-ppla and pplab. Ribosomal frameshifting takes place during the translation of ORF1a to synthesize pplab.

Viral-derived proteases, such as, 3-chymotrypsin-like protease and papain-like protease, which are synthesized through the translation of viral genomic (+) RNA, cause the proteolysis of pp1a and pp1ab to generate nsp 1-16. The nsp 1-16, and other proteins of the RTC, including Replicase and Helicase accumulate at the perinuclear regions and assemble to form double membranebound RTC. Hydrophobic regions present in nsp 3, 4, and 6 adhere the nascent pp1a/pp1ab to the membrane during the initial stages of RTC formation.

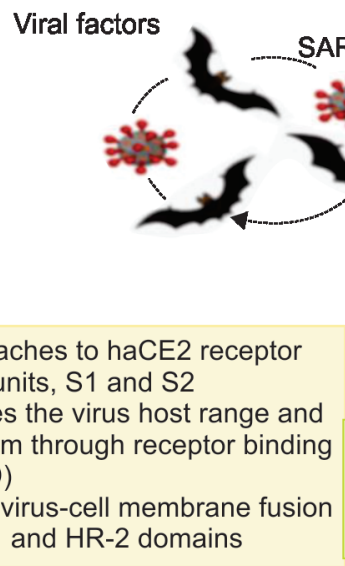

S protein-it attaches to haCE2 receptor through 2 subunits, S1 and S2

- $\mathrm{S} 1$ determines the virus host range and cellular tropism through receptor binding domain (RBD)

- S2 mediates virus-cell membrane fusion through HR-1 and HR-2 domains
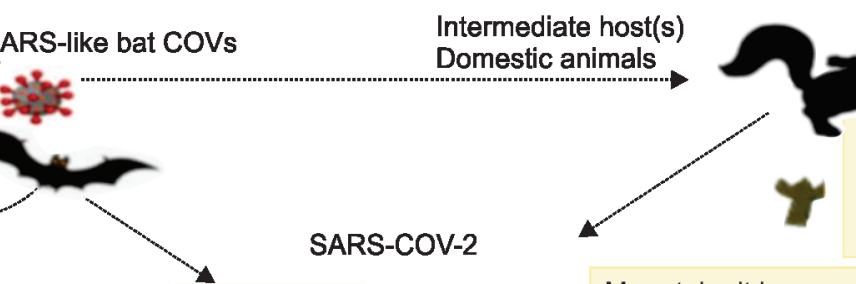

Bat- ACE-2 binding present Swine- ACE-2 binding present Civet- ACE-2 binding present Mouse- ACE-2 binding absent

SARS-COV-2

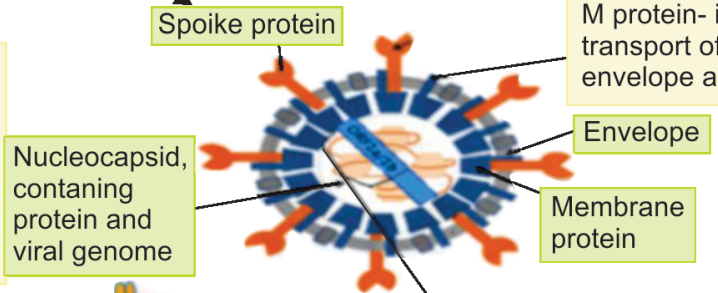

$M$ protein- it is responsible of the transmembrable transport of nutrients, the formation of the viral and the release of bud

$\mathrm{N}$ and $\mathrm{E}$ proteins-they interfere with host immune response
SARS-COV-2 receptor: human angiotensin converting enzyme 2 (haCE2)

Persons susceptible to severe disease - Elderly (more than 65 years old)

- People with co-morbidities

Cytokine storm leading to sustained inflammatory responses, hypoxia, and septic shock are some of the critical conditions of SARAS-COV-2 infected patients
16 non-structure proteins: nsp1-nsp16

Underlying health conditions that increases susceptibility COVID-19 - Hypertension

- Diabetes mellitus

- Cardiovascular diseases

Severe complication of COVID-19

- Acute respiratory disress syndrome (ARDS)

- Septic shock

- Metabolic acidosis

- Dysfunction of coagulation

- Multiple organ failure

Fig. 1: Overview of infection and pathology of COVID-19 ${ }^{3}$ 


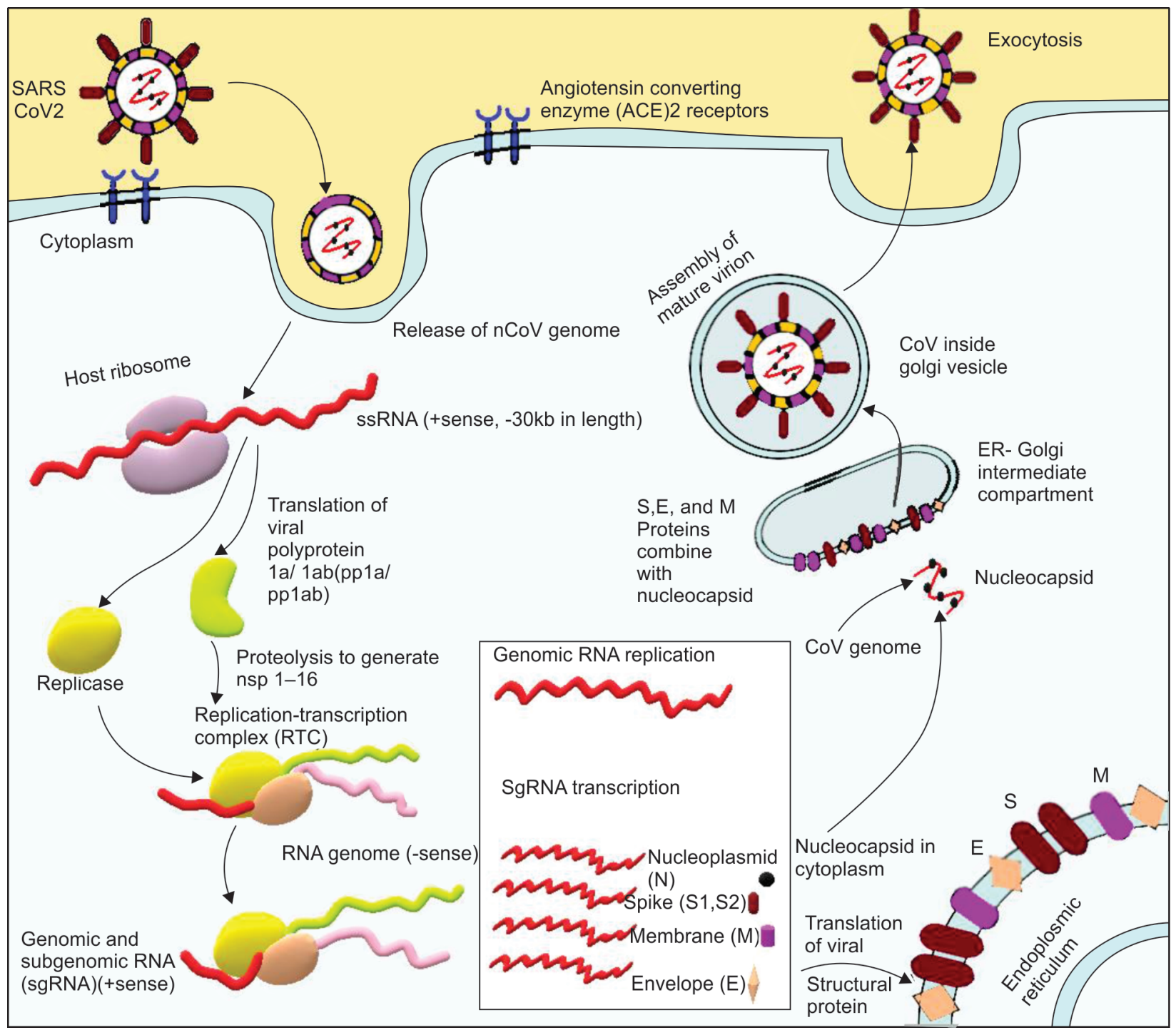

Fig. 2: SARS-CoV-2 entry, replication, and release

The RTC first replicates the entire SARS-CoV-2 genomic (+) RNA to form a full length, antisense (-) RNA template. This RNA template is then used for transcription by the RTC to synthesize:

- Severe acute respiratory syndrome-coronavirus-2 genomic (+) RNA.

- Subgenomic mRNA, i.e., (+) sgRNA encoding viral structural proteins $-S, M, N$, and $E$ proteins, synthesized through the process of discontinuous transcription.

The (+) sgRNA is then translated to form viral structural proteins-S, M, N, and E proteins. Assembly of the SARS-CoV-2 virion takes place in vesicles. These vesicles undergo fusion with the cell membrane, and the SARS-CoV-2 virions are released by exocytosis. The newly synthesized SARS-CoV-2 (+) genomic RNA is used for further synthesis of SARS-CoV-2 virions.

\section{Host Response to COVID-19}

\section{Innate Immune Response}

Active viral replication and release in SARS-CoV-2 infected cells cause the cells to undergo pyroptosis, which is an extremely inflammatory form of apoptosis. This causes the release of viral RNA and damage-associated molecular patterns (DAMPs), such as, ATP, DNA, and apoptosis-associated speck-like protein like oligomers (ASC-like oligomers). These are recognized by various pattern recognition receptors (PRRs) on airway epithelial cells, cells of the vascular endothelium, as well as alveolar macrophages. This causes increased expression and release of proinflammatory cytokines including IL-6, interferon-gamma (IFNY), MCP1, IP-10, and MIP1a. These precipitate local inflammation in the lungs, and also cause a severe influx of monocytes/macrophages, dendritic cells, and T lymphocytes (but not neutrophils) into the lower respiratory tract from the blood, exacerbating the inflammatory immune response. Particularly, pulmonary recruitment and infiltration of highly inflammatory $\mathrm{FCN1}^{+}$macrophages take place in COVID-19. The innate immune response is primarily responsible for the cytokine storm in severe cases of COVID-19.

\section{Phagocytosis (Primarily by Dendritic Cells in COVID-19)}

Severe acute respiratory syndrome-coronavirus-2 has specific pathogen-associated molecular patterns (PAMPs) on its viral 
envelope which are recognized by specific PRRs on the cell membrane of dendritic cells and macrophages (to a less extent), while the virus is in the extracellular domain before they have infected alveolar type II pneumocytes. This induces phagocytosis of the SARS-CoV-2 pathogens and their internalization in a phagosome. Once internalized, SARS-CoV-2 PAMPs in the form of viral genomic RNA or the intermediates during viral replication including dsRNA, is recognized by specific endosomal and cytoplasmic PRRs including.

- Endosomal RNA receptors - toll-like receptors (TLR) - TLR3 and TLR7. TLR3 is expressed in dendritic cells, while TLR7 is expressed in dendritic cells and monocytes/macrophages including dust cells present in the lungs.

- Cytoplasmic RNA sensors-retinoic acid-inducible gene I (RIG-I), and melanoma differentiation-associated gene 5 (MDA5).

The phagosomes then fuse with the lysosomes to form phagolysosomes. ${ }^{5}$ Phagolysosomes use several mechanisms for the destruction of the infected cells, which include:

- Lysosomal acid hydrolases, such as, nucleases, lipases, and proteases.
- Several oxygen radicals, such as, superoxide radicals $\left(\mathrm{O}_{2}^{-}\right)$, hypochlorite $\left(\mathrm{HOCl}^{-}\right)$, hydrogen peroxide $\left(\mathrm{H}_{2} \mathrm{O}_{2}\right)$, and hydroxyl radicals $\left(\mathrm{OH}^{\circ}\right)$, are extremely toxic to microbes. The action of these oxygen radicals involves transient highly increased oxygen uptake (oxidative burst).

The recognition of the viral RNA genome and other virusderived RNA products by TLR3, TLR7, and RIG-1/MDA5 receptors on dendritic cells activates a downstream signaling cascade in the dendritic cell by binding to the mitochondrial antiviralsignaling protein (MAVS), activating the transcription factors NF-KB and interferon regulatory factor 3 (IRF3), and causing their translocation to the nucleus. Usually, activation of TLR3/7 induces the translocation to the nucleus of NF-kB, while activation of RIG-1/ MDA5 results in the nuclear translocation of IRF3.

In the nuclei, these transcription factors induce the transcription, synthesis, as well as secretion of type I interferon (IFNa), through IRF3, and other proinflammatory cytokines including IL-1 $\beta$, IL-6, and TNF- $a$, through NF-KB. IL-8, IL-12, and IL-23 are also secreted. In addition, in COVID-19, type II IFN (IFN- $\gamma$ ) is also produced by natural killer (NK) cells, $C D 4^{+} \mathrm{T}_{\mathrm{h}} 1$ and $\mathrm{CD} 8^{+}$cytotoxic $\mathrm{T}$ lymphocyte (CTL) effector T cells.
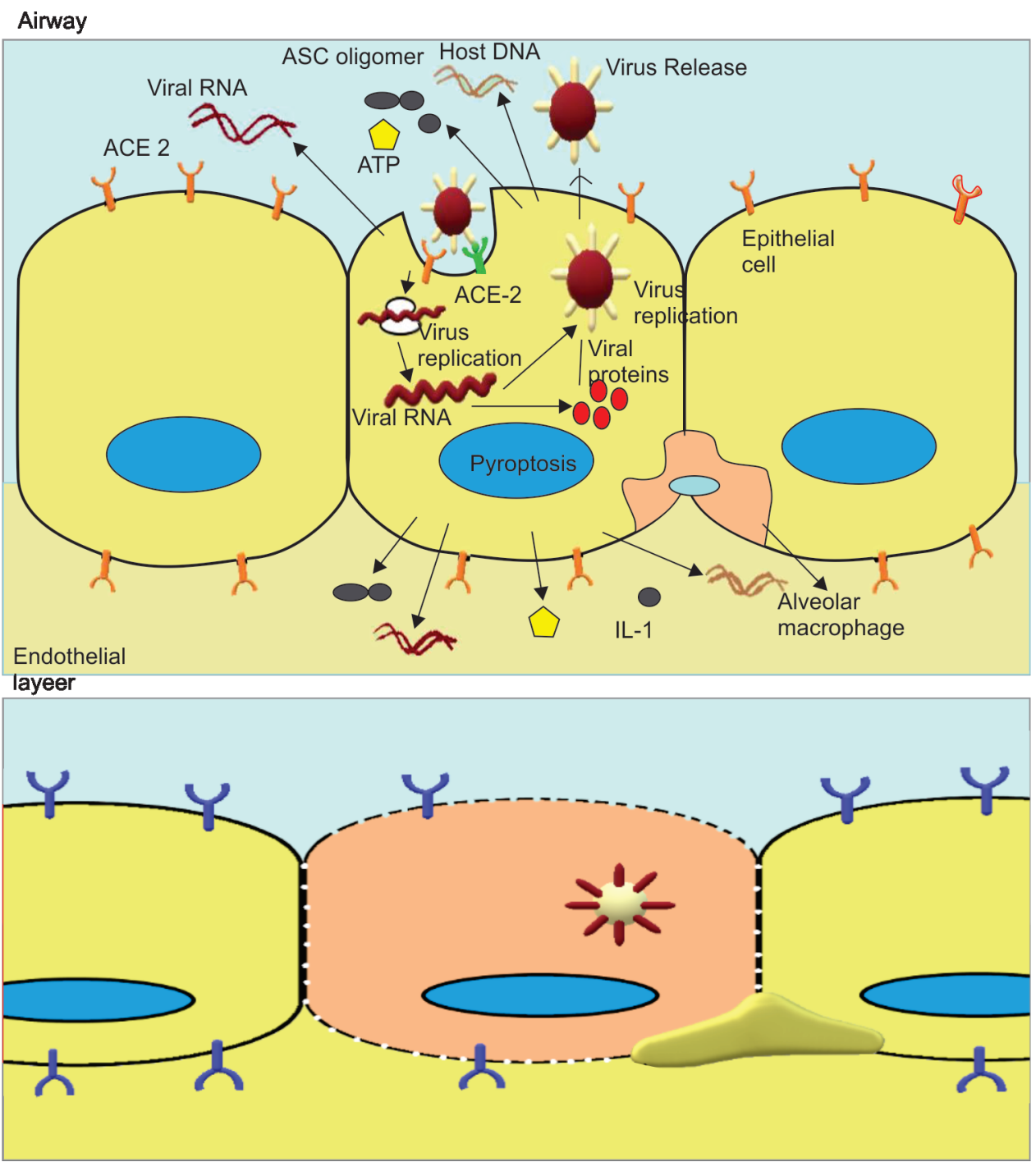


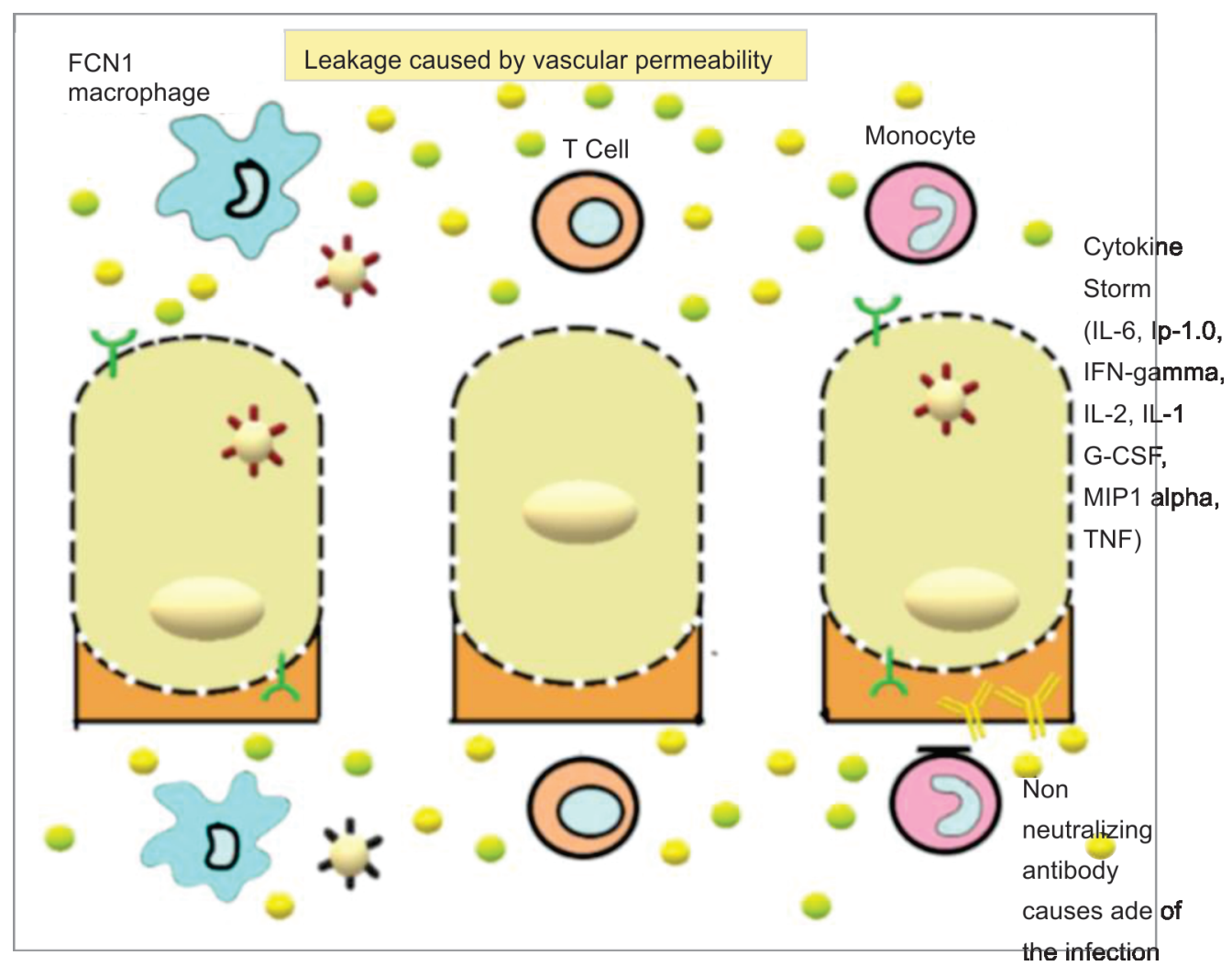

Fig. 3: Overview of an innate immune response to SARS-CoV- $2^{4}$

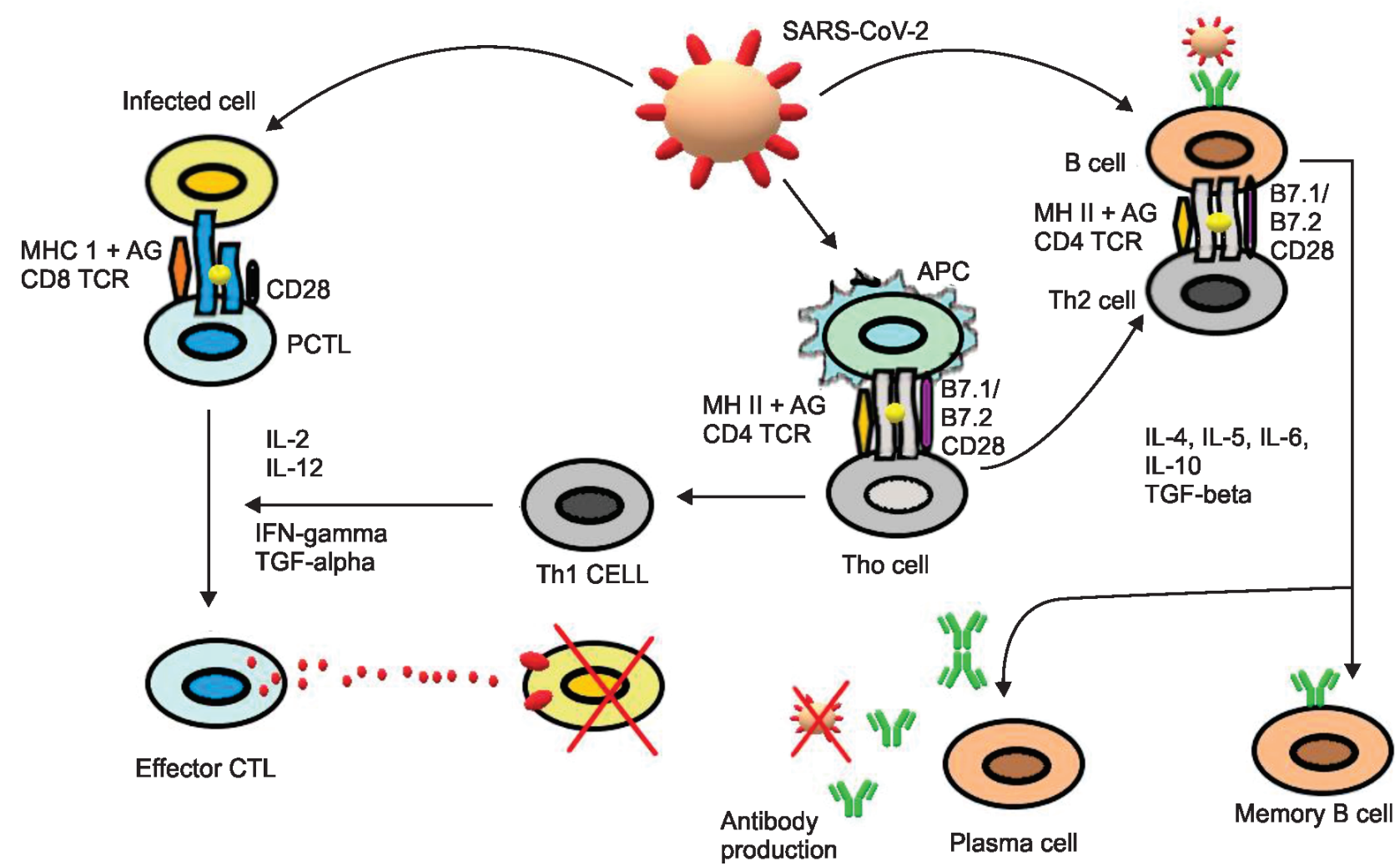

Fig. 4: Overview of an adaptive immune response to SARS-CoV-2. CD, cluster of differentiation; TCR, T cell receptor; AG, antigen 


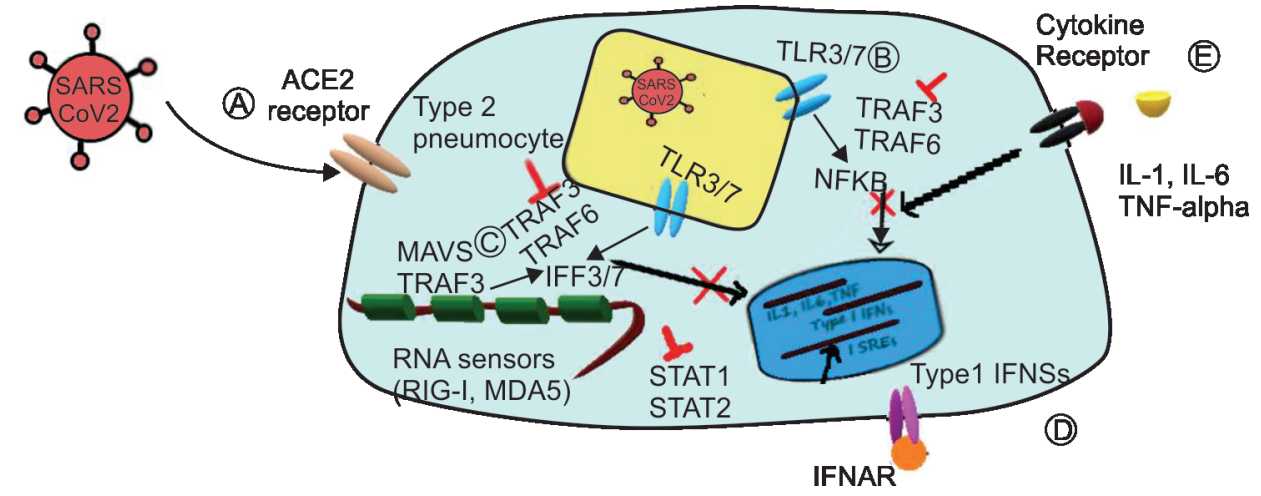

Fig. 5: Mechanism and sites of immune evasion in SARS-CoV-2 infected cells. ${ }^{6}$ (Sites of immune evasion are shown in red.) MAVS, mitochondrial antiviral signaling protein; IFNAR, receptor for type I IFN; ISREs, IFN stimulated response elements

\begin{tabular}{ll}
\hline Cytokine/chemokine & Actions \\
\hline Type I interferon (IFNa) & Stimulates dendritic cells, natural \\
killer cells, and macrophages to elic- \\
it an antiviral response comprising \\
of RNAdependent protein kinase \\
(PKR) as well as apoptotic pathways, \\
through the IRF3/7 pathways. \\
Induces fever by binding to opioid \\
receptors in the hypothalamus, and \\
eliciting the release of prostaglan- \\
din- $\mathrm{E}_{2}\left(\mathrm{PGE}_{2}\right)$.
\end{tabular}

Type II interferon (IFN- $\gamma$ ) Promotes NK cell and macrophage activity.

Increases cell surface $\mathrm{MHC}$ I and MHC Il expression in antigenpresenting cells (APCs), enhancing antigenic presentation, and consequently the adaptive immune response.

Induces class shifting from IgM to $\lg G$.

Interleukin-1 $\beta$ (IL-1 $\beta)$

Interleukin-6 (IL-6)

Interleukin-8 (IL-8), a chemokine

Interleukin-12 (IL-12)

Interleukin-23 (IL-23)

Tumor necrosis factor-a (TNF-a)
These substances promote inflammation, fever, and the chemotaxis of dendritic cells, macrophages, and NK cells, to the infection site, i.e., the alveoli.

\section{Complement System}

In COVID-19, the complement system does not directly lead to the destruction of SARS-CoV-2, as the virus has no cell membrane and therefore formation of the membrane attack complex (MAC), initiated by C5b has little effect in causing the lysis of SARS-CoV-2.

Furthermore, as SARS-CoV-2 is intracellular for much of its life cycle, and parasitizes alveolar type II pneumocytes, it makes it even more difficult for the complement system to detect the virus in the extracellular domain.

Nevertheless, the complement system, especially the anaphylatoxins $\mathrm{C} 3 \mathrm{a}, \mathrm{C} 4 \mathrm{a}$, and $\mathrm{C} 5 \mathrm{a}$, plays a role in the chemotaxis and activation of NK cells, dendritic cells, neutrophils, and macrophages to the infection site, which contributes to the cytokine storm and ARDS in severe cases.

\section{Natural Killer Cells}

IL-12 and IFNa secreted by activated dendritic cells cause chemotaxis of NK cells to the infection site as well as causing their activation.

Nucleated cells, including alveolar type II pneumocytes, have cell surface MHC class I expression. Once infected by SARS-CoV-2 these alveolar type II pneumocytes respond by increasing cell surface expression of stress-associated molecules, including MICA and MICB. ${ }^{5}$ Severe acute respiratory syndrome-coronavirus- 2 also causes downregulation of cell surface expression of MHC class I molecules. This results in a reduction in the number of MHC-I proteins on the cell surface. Killer activation receptors (KARs) present on the surface of NK cells recognize and bind to MICA and MICB on the cell surface membrane of infected alveolar type II pneumocytes. This provides a signal for destruction, i.e., a "kill signal". Killer inhibition receptors (KIRs) present on the surface of NK cells recognize and bind to MHC-I proteins on the cell surface of the infected cells. As there is insufficient binding between KIRs and MHC-I proteins on the infected alveolar type II pneumocytes, due to SARS-CoV-2 induced MHC-I downregulation, therefore KIRs do not provide a signal to override the "kill signal" produced by KAR-MICA/MICB binding.

As insufficient KIR-MHC I binding occurs due to downregulation of $\mathrm{MHC}$ I expression, KIRs do not override the KAR kill signal. ${ }^{5}$

As a result, this leads to the lysis and destruction of infected alveolar type Il pneumocytes by cytotoxic perforins and granzymes 


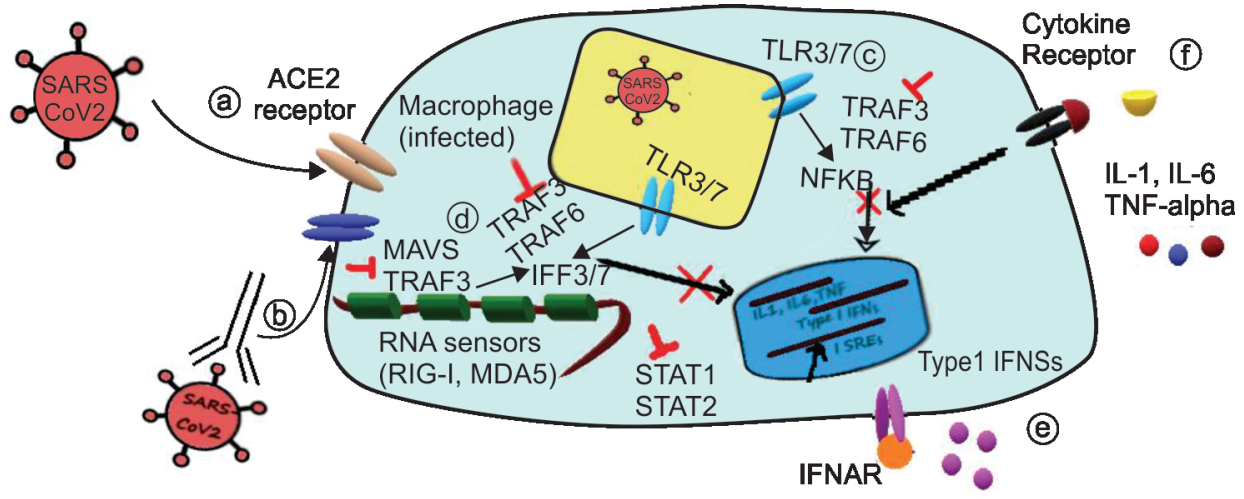

Fig. 6: Mechanism of immune evasion, inhibition of IFN-1 pathway, and antibody-directed enhancement (ADE) in SARS-CoV-2 infected macrophages ${ }^{6}$

exocytosed by NK cells. This is one of the major causes of lung damage and ARDS.

\section{Adaptive Immunity \\ Antigen Presentation}

In COVID-19, antigenic presentation primarily through MHC-I complexes, as SARS-CoV-2 is essentially an intracellular virus. However, the antigenic presentation also occurs minorly through MHC-Il complexes.

\section{Presentation by MHC Class II (for Extracellular/ Exogenous SARS-CoV-2 Antigens)}

- When the SARS-CoV-2 pathogens have not yet infected alveolar type II pneumocytes, and are still in the extracellular domain, they are phagocytized by dendritic cells through clathrinmediated actin-dependent endocytosis or clathrin-independent macropinocytosis.

- There is a reduction in the phagocytic activity, and an increase in the synthesis and cell surface expression of MHC-II proteins in dendritic cells, which subsequently migrate to lymph nodes.

- The pMHC-II complex is displayed on the cell surface of the dendritic cells, for potential recognition by MHC-II restricted $\mathrm{CD} 4^{+} \mathrm{T}_{\mathrm{h}}$ cells, which have specific $\mathrm{T}$ cell receptors (TCRs).

\section{Presentation by MHC Class I (for Intracellular/ Endogenous SARS-CoV-2 Antigens)}

- Once the SARS-CoV-2 pathogens have infected the alveolar type II pneumocytes, cytoplasmic proteins of self as well as the non-self origin, including cytoplasmic SARS-CoV-2 are covalently attached to ubiquitin. As a result, these proteins are targeted for proteolytic degradation by the proteasome enzyme complex.

- Proteasome-generated peptide fragments bind to MHC-I on the cell surface.

\section{Adaptive Immune Response}

In COVID-19, SARS-CoV-2 antigen-specific CD4 ${ }^{+} \mathrm{T}$ cells primarily express IFN $\gamma$, TNF, and IL-2. ${ }^{4}$ This suggests that in patients infected with SARS-CoV-2, the $T_{h} 1$ response predominates, and therefore the cell-mediated immune response is primarily responsible for viral clearance.

\section{Cell-Mediated Immune Response}

Cell-mediated responses comprise two basic forms:

- Cell-mediated lysis, mediated by $C D 8^{+}$CTLs.

- Delayed type hypersensitivity (DTH), mediated by $C D 4^{+} T_{h} 1$ cells.

\section{Immunological Synapse}

There is weak interaction between the $\mathrm{CD}^{+} \mathrm{TCR}$ and the MHC-IISARS-CoV-2 antigen complex on dendritic cells, and between the CD8 ${ }^{+}$TCR and the MHC-I-SARS-CoV-2 antigen complex on alveolar type II pneumocytes. CD4/CD8 molecules binding to the "constant" non-peptide-binding portions of pMHC stabilize this interaction. This constitutes the TCR signal. The TCR signal, a costimulatory signal, and a cytokine signal are necessary for the differentiation, activation, and proliferation of both $\mathrm{CD} 4^{+}$and $\mathrm{CD} 8^{+} \mathrm{T}$ cells.

\section{Activation of $\mathrm{CD}^{+} \mathrm{T}$ Cells}

Naïve $C D 4^{+}$T-helper precursor $\left(T_{h p}\right)$ cells become activated after the immunological synapse in which the TCR recognizes MHCII-SARS-CoV-2 antigen complex on dendritic cells (APC). The $\mathrm{CD} 4^{+} \mathrm{T}$-cells are stimulated to secrete IL-2 and express receptors for IL-2 on its cell membrane, forming pathway-uncommitted $\mathrm{T}_{\mathrm{h}} 0$ cells.

Since the levels of IFN- $\gamma$ secreted by activated NK cells and IL-12 secreted by activated dendritic cells are very high in COVID-19, therefore the $T_{h} 0$ primarily mature along the $T_{h} 1$ functional pathway to differentiate to become $T_{h} 1$ cells, which activate cytotoxic $C D 8^{+}$ T cells (CTL), and therefore the cell-mediated response dominates in COVID-19.

Some $T_{h} 0$ cells also differentiate to form $T_{h} 2$ that activate B-lymphocytes responsible for the humoral response.

$T_{h} 1$ cells secrete IFN $\gamma$, which in turn causes more undifferentiated $C D 4^{+}$cells $\left(T_{h} 0\right.$ cells) to differentiate into $T_{h} 1$ cells representing a positive feedback loop while suppressing $T_{h} 2$ cell differentiation. IL-12 secreted by activated dendritic cells, and IL-10 secreted by activated macrophages also increases $T_{h} 1$ differentiation and suppresses $T_{h} 2$ differentiation. This enhances cell-mediated immune response and downregulates the humoral immune response. (Thus, convalescent plasma of recovered COVID patients is being used as a means of strengthening humoral immunity in COVID patients, as cell-mediated immunity is one of the major causes of ARDS.) 


\section{$\mathrm{T}_{\mathrm{h}} 17$ Cells}

IL-6 and IL-23 secreted by activated dendritic cells contribute to the differentiation of $T_{h} 0$ cells into $T_{h} 17$ cells, which not only augment the $T_{h} 1$ response, but they also promote inflammatory events in various tissues. They secrete IL-17, which acts on neutrophils and dendritic cells, attracting them to sites of inflammation. IL-17 induces these cells to produce proinflammatory cytokines, IL-1, IL-6, transforming growth factor-13 (TGF-13), and G-CSF, and the chemokine, IL-8. This contributes to the cytokine storm and ARDS in severe COVID cases.

\section{DTH Response}

Activated $C D 4^{+} T_{h} 1$ cells activate macrophages by direct contact (that involves the binding of CD40 with CD154) as well as by IFN- $\gamma$ secreted by $T_{h} 1$ cells. The activated macrophages are not antigenspecific and destroy infected type II pneumocytes as well as surrounding lung tissue indiscriminately, through phagocytosis, hydrolytic enzymes, and reactive oxygen species.

\section{Activation of $\mathrm{CD}^{+} \mathrm{T}$ Cells}

Recognition of MHC-I-SARS-CoV-2 antigen complex on the surface of infected alveolar type II pneumocytes/dendritic cells by specific TCR of naïve $C D 8^{+}$T-cells causes them to increase the expression of IL-2 receptors.

- Activated CD4 ${ }^{+} \mathrm{T}_{\mathrm{h}} 1$ cells secrete IL-2, which binds to IL-2 receptors on the surface of $C D 8^{+} T$-cells ( $T_{c}$ cells).

- Interaction between the APC and the $\mathrm{CD} 4^{+} \mathrm{T}$ cell increases the expression of CD80/86 by the APC (dendritic cell).

- Interaction between the APC CD8O/86 and the CD28 on CD8 ${ }^{+}$ T-cells promotes $\mathrm{CD} 8^{+} \mathrm{T}$-cell differentiation and proliferation into CTLs.

\section{Cell-Mediated Lysis}

Completely differentiated CTLs secrete perforin, a cytolytic protein, as well as granzyme. These substances induce apoptosis of the infected target cell expressing the specific pMHC-I complex, i.e., the infected alveolar type II pneumocyte or dendritic cell. Engagement of FasL on CTL with Fas on target cell initiates Fas-induced apoptosis (extrinsic pathway) of a target cell.

\section{Memory Cell Generation}

In the majority of stimulated CD4 ${ }^{+}$T cells, CD 152 (cytotoxic T lymphocyte-associated antigen-4 or CTLA-4), which is normally sequestered inside the Golgi apparatus, is transported to the cell membrane, where it binds to CD80/86. ${ }^{5}$ Binding between CD152 and CD80/86 inhibits T cell IL-2 mRNA expression and stops cell proliferation. This ensures the self-limiting nature of $\mathrm{CD}^{+}{ }^{+} \mathrm{T}$ cellmediated responses.

Some $\mathrm{CD}^{+}{ }^{+} \mathrm{T}$ cells, however, form memory cells. Memory $\mathrm{T}$ cells increase the expression of CD28, and this makes them more likely to rapidly respond to CD80/86 expressed by APCs in SARS$\mathrm{CoV}-2$ infection. Memory cells also decrease the surface expression of CD62L (L-selectin). This causes memory T cells to travel to the infection site (lungs) for potential subsequent infection by SARSCoV-2.

Stimulated T cells also secrete certain cytokines.

- IFN- $\gamma$ is secreted by both $C D 4^{+} T_{h} 1$ and CD8 ${ }^{+}$CTL cells. It promotes the differentiation of $C D 4^{+}$cells $\left(T_{h} 0\right.$ cells) into $T_{h} 1$ cells, enhancing cell-mediated immunity while suppressing $T_{h} 2$ cell differentiation, which downregulates the humoral response, which is characteristic of COVID-19.

- Tumor necrosis factor-a secreted by both $C D 4^{+} \mathrm{T}_{\mathrm{h}} 1$ and $\mathrm{CD} 8^{+}$ CTLs, which increases vascular permeability.

- IL-2, secreted by CD4 $4^{+} T_{h} 1$ cells, which activates $T_{c}$ cells to form CTLs.

\section{Humoral Response}

In COVID-19, the humoral response is largely downregulated due to the suppression of $T_{h} 2$ differentiation as a result of IFN- $\gamma$, IL-10, and IL-12. However, it does take place and is mediated by B-lymphocytes. Furthermore, as SARS-CoV-2 is primarily intracellular, humoral responses have little effect on it. B-lymphocyte responses normally occur concomitantly with the $T_{h}$ lymphocyte responses. In the initial stages of infection, particularly in the first week, non-neutralizing antibodies are produced against the nucleocapsid $(\mathrm{N})$ protein. Neutralizing antibodies are produced against the spike $(S)$ protein by the 2 nd to 3 rd week of SARS-CoV-2 infection.

\section{Activation and Action of B-Lymphocytes}

In COVID-19, B-lymphocytes are primarily activated through the T-cell-dependent mechanism.

- Direct contact between the B-cell receptors (BCRs) of undifferentiated B-lymphocytes with the SARS-CoV-2 specific TCR on T-cells causes the B-lymphocytes to express IL-4 receptors on their cell surface.

- This direct contact, along with the costimulation through CD28CD80/86 interaction and/or CD40-CD154 interaction, activates the B-lymphocyte.

- In the presence of IL-4, which is secreted by stimulated macrophages, certain $T_{h} 0$ cells differentiate to form $T_{h} 2$ cells. $\mathrm{T}_{\mathrm{h}} 2$ cells secrete IL-4, and this IL-4 binds to IL-4 receptors on B-cells. This causes the proliferation of B cells as well as differentiation of B-lymphocytes into plasma cells. Plasma cells, in turn, secrete SARS-CoV-2-specific IgM antibodies in the initial phase of infection and subsequently SARS-CoV-2-specific IgG antibodies. The antibody class shift from IgM to lgG is induced by IFN- $\gamma$ and IL-10.

- IgG antibodies bind to SARS-CoV-2 antigens in the extracellular domain and indirectly cause the agglutination or neutralization of the SARS-CoV-2 virions. This makes them more susceptible to phagocytosis.

- IL-4 causes the class to shift from IgM to IgE. The Fc region of SARS-CoV-2-specific IgE becomes bound to cell surface receptors on basophils and mast cells. When these lgE antibodies encounter a SARS-CoV-2 epitope, they degranulate releasing histamine, leukotrienes, and cytokines which causes type I hypersensitivity reactions leading to bronchoconstriction, hypermotility of intestines, vasodilation, and increased vascular permeability leading to symptoms of COVID including rashes, breathlessness, hypotension, diarrhea, etc.

\section{Immune Evasion by SARS-CoV-2}

In the initial stages of COVID-19 infection, SARS-CoV-2 can suppress the IFN-I pathway induced by IFN-a, leading to the delayed transcription of IFN stimulated genes, and the delayed activation of the innate immune response, including the release of inflammatory cytokines and infiltration of phagocytic cells which produce symptoms. This allows the unrestricted replication, proliferation, and spread of SARS-CoV-2 through lung tissue. This is the reason 
why COVID-19 does not produce symptoms in the initial days of the infection, and the reason why asymptomatic infected individuals can transmit the pathogen. This suppression may be achieved through the following means:

- Severe acute respiratory syndrome-coronavirus-2 induces the formation of double-membrane bound vesicles, which do not have PRRs and subsequently replicate within these vesicles. This prevents the detection of SARS-CoV-2 dsRNA by toll-like receptors and RIG-I/MDA5 receptors of dendritic cells/ macrophages.

- Severe acute respiratory syndrome-coronavirus-2 suppresses the activation of TNF receptor-associated factors (TRAF) 3 and 6 , and thus inhibits the activation of the transcription factors NFKB and IRF3 and $7 .{ }^{6}$ At the step of IFN-I induction, SARS-CoV-2 causes the ubiquitination and degradation of MAVS and inhibits IRF3 nuclear translocation. This prevents the transcription, synthesis, and secretion of IFN-a. This suppresses the initial proinflammatory response induced by type I IFN and the proinflammatory cytokines IL-1, IL-6, and TNF-a.

- Once IFN-a has been secreted, SARS-CoV-2 inhibits the IFN-I induced JAK-STAT pathway by decreasing the phosphorylation of STAT-I. Thus, the activation of STAT transcription factors, in response to the activation of type I IFN receptors, is inhibited. ${ }^{6}$

- Tissue macrophages and monocytes have very low levels of ACE-2 expression. As a result, SARS-CoV-2 infection through $A C E-2$ receptor interaction is very limited. However, ineffective non-neutralizing antibodies produced by B-lymphocytes in the initial stages of infection can bind to SARS-CoV-2 forming immune complexes. These immune complexes are endocytosed by macrophages through Fcy receptors, which leads to viral infection of macrophages. This process is called antibodydirected enhancement (ADE). Persistent viral infection in macrophages results in inhibition of type IIFN-induced signaling pathways, and aberrant production of proinflammatory cytokines, particularly IL-1 $\beta$, IL-6, and TNF- $\alpha$.

The host immune response against SARS-CoV-2 relies heavily on the type I IFN pathway. Therefore, inhibition of the IFN-I pathway hinders viral clearance and aids viral replication. The reduced IFN-a secretion causes a reduction in initiation of antiviral protective mechanisms to contain the spread of SARS-CoV-2, and thus the virus proliferates even more. This results in the increased release of products of pyroptosis and exacerbates aberrant inflammatory responses. Furthermore, SARS-CoV-2 induces the apoptosis of T-cells, thus making viral clearance by the adaptive immune response more difficult.

\section{Cytokine Storm}

Cytokine storm is the deadliest feature of COVID-19. It is the uncontrolled systemic inflammatory response as a result of the release of excess amounts of proinflammatory cytokines, such as TNF- $\alpha$, IL-1, IL-6, IL-12, IFN- $\alpha$, IFN- $\beta$, IFN- $\gamma$, and IL- 8 , by activated cells primarily belonging to the innate immune system including dendritic cells, macrophages, and neutrophils, as well as cells of the adaptive immune system, primarily T lymphocytes.

- IL-17 activates $T_{h} 17$ cells, which augment the $T_{h} 1$ response, leading to an exaggerated cytotoxic $T$ cell response, and cause neutrophil chemotaxis and activation to the site of infection.

- Macrophages receive activating signals through type IIFN (IFN$a / \beta$ ) receptors on their surface, and produce more monocyte/ macrophage chemoattractants (such as, CCL2, CCL7, and CCL12), resulting in the further chemotaxis and accumulation of macrophages. These macrophages produce TNF-a, IL-6, and IL-1 $\beta .^{7}$

The net effect of the cytokine storm is to cause severe inflammation in the sites of infection as well as immense infiltration of phagocytic cells, primarily neutrophils, and macrophages, as well as CTLs to the site of infection, i.e., lungs. It also causes an overactivation of Tlymphocytes, which is manifested by an increase in $T_{h} 17$ and the high cytotoxicity of $\mathrm{CD}^{+} \mathrm{T}$ cells.

Furthermore, IFN- $a$ as well as some macrophage-derived cytokines induce the apoptosis of $\mathrm{T}$ cells, resulting in low peripheral levels of $C D 4^{+} T_{h} 1$ and $C D 8^{+} C T L$ cells. This not only hinders viral clearance allowing the uncontrolled replication and spread of SARSCoV-2 but also causes the non-antigen specific macrophage and neutrophil-mediated phagocytic response to predominate over the SARS-CoV-2 antigen-specific CTL-mediated response, causing a large-scale non-specific lung tissue damage.

\section{Disease Spectrum}

- Most patients (80\%) with COVID-19 develop mild to moderate symptoms. Approximately 15\% develop severe pneumonia, and $5 \%$ progress to develop ARDS. ${ }^{1}$ Patients with underlying comorbidities, such as, cardiovascular disease, diabetes mellitus, renal disease, or chronic pulmonary disease, are at a higher risk of developing severe disease.

- Nausea, vomiting, and diarrhea result due to SARS-CoV-2 infection of the small intestinal cells (having ACE-2) and the increased intestinal motility as a result of the type I hypersensitivity response.

- Large-scale pulmonary recruitment of lymphocytes from the blood and not neutrophils is responsible for the lymphopenia and the high neutrophil-lymphocyte ratio seen in the blood of COVID-19 patients.

- Massive infiltration of cytotoxic T cells, but more importantly non-antigen specific macrophages and neutrophils to the site of infection, i.e., lungs destroys both infected and non-infected alveolar type I and surfactant producing type II pneumocytes. The destruction of alveolar type II pneumocytes results in a decrease in the surfactant level in the lungs and consequently, increases surface tension. This reduces the ability of the lungs to expand and therefore increases the risk of lung collapse and respiratory failure.

- Massive pulmonary recruitment and pathological hyperinflammatory responses by immune effector cells lead to immune exhaustion in COVID-19, indicated by upregulation of exhaustion markers, such as, NKG2A, on CD8 ${ }^{+}$CTLs and NK cells. This immune exhaustion further hinders viral clearance.

- IFN- $\alpha$ and IFN- $\gamma$ induce the apoptosis of epithelial cells in the alveoli and the lower respiratory tract through mechanisms, that involve the Fas-Fas ligand (FasL) or TRAIL-death receptor 5 (DR5). ${ }^{7}$ This causes alveolar damage with the formation of hyaline membranes. This damages the pulmonary microvascular and alveolar epithelial cell barriers and consequently causes vascular leakage and alveolar edema. This severely compromises alveolar gas exchange, leading to hypoxia. It also makes the lung more vulnerable to secondary bacterial or fungal infections.

- In COVID-19, lungs are filled with clear liquid jelly. This is suspected to be hyaluronic acid (HA). In COVID-19, the 
production and regulation of $\mathrm{HA}$ are defective. This is because the high levels of cytokines IL-1, and TNF in the lungs as a result of the cytokine storm are strong inducers of HA-synthase-2 in endothelial cells, lung alveolar epithelial cells, and fibroblasts. Hyaluronic acid can absorb water up to 1,000 times its molecular weight, and therefore causes severe edema in the lungs.

- Severe acute respiratory syndrome-coronavirus-2 downregulates ACE-2 expression in infected cells in the alveoli and the lower respiratory tract. Angiotensin-converting enzyme-2 is responsible for the regulation of the renin-angiotensinaldosterone-system (RAAS). Therefore, loss of pulmonary ACE-2 function, which is associated with acute lung injury in COVID19 , results in dysfunction of the RAAS. ${ }^{4}$ This leads to severe dysregulation of blood pressure, and electrolyte imbalance. It also leads to an increase in vascular permeability, enhancing inflammation in the airways.

- Severe acute respiratory syndrome-coronavirus-2 infection of cardiac cells and vascular endothelia is responsible for cardiovascular morbidities in severe cases of COVID-19, which include tachyarrhythmia, myocardial infarcts (indicated by elevated cardiac troponin-T levels), as well as venous thromboembolic events. These are associated with high mortality risk. Inflammation in COVID-19 results in severe dysregulation of negative feedback loops that inhibit thrombin generation through antithrombin III and the protein-C system. Uncontrolled thrombin generation increases thrombininduced platelet aggregation through proteinase-activated receptor-1 (PAR-1). This increases the risk of microthrombosis and disseminated intravascular coagulation. Therefore, patients with severe COVID-19 are predisposed to develop venous thromboembolism, which is indicated by elevated levels of d-dimer in the blood of patients with severe disease. ${ }^{8}$

- High levels of proinflammatory cytokines may lead to shock and tissue damage in the heart (which may lead to circulatory failure), liver, and kidney. Spleen atrophy and lymph node necrosis are also observed. This leads to multiple organ failure and even death in very severe cases of COVID-19.

\section{Treatment}

Clinical treatment primarily includes symptomatic management and oxygen therapy. A multitude of drugs-antiviral, hydroxychloroquine, anti-IL-6 are also being used. The role of plasma therapy is still controversial. Mechanical ventilation is needed for patients who develop ARDS. ${ }^{1}$

\section{References}

1. Xuetao C. COVID-19: immunopathology and its implications for therapy. Nat Rev Immunol 2020;20(5):1-2. DOI: 10.1038/s41577-0200308-3.

2. Shi $H, \operatorname{Han} X$, Jiang N, et al. Radiological findings from 81 patients with COVID-19 pneumonia in Wuhan, China: a descriptive study. Lancet Infect Dis 2020;20(4):425-434. DOI: 10.1016/S1473-3099(20)30086-4.

3. Guo YR, Cao QD, Hong ZS, et al. The origin, transmission and clinical therapies on coronavirus disease 2019 (COVID-19) outbreak-an update on the status. Mil Med Res 2020;7(1):11. DOI: 10.1186/s40779020-00240-0PMID: 32169119; PMCID: PMC7068984.

4. Tay MZ, Poh CM, Rénia L, et al. The trinity of COVID-19: immunity, inflammation and intervention. Nat Rev Immunol 2020;20(6):363-374. DOI: 10.1038/s41577-020-0311-8.

5. Doan T, Melvold R, Viselli S, et al. Lippincott's Illustrated Reviews: Immunology. Philadelphia: Wolters Kluwer Health; 2013.

6. Felsenstein S, Herbert JA, McNamara PS, et al. COVID-19: immunology and treatment options. Clin Immunol 2020;215:108448. DOI: 10.1016/j. clim.2020.108448Epub 2020 Apr 27. PMID: 32353634; PMCID: PMC7185015.

7. Channappanavar R, Fehr AR, Vijay R, et al. Dysregulated type I interferon and inflammatory monocyte-macrophage responses cause lethal pneumonia in SARS-CoV-infected mice. Cell Host Microbe 2016;19(2):181-193. DOI: 10.1016/j.chom.2016.01.007PMID: 26867177; PMCID: PMC4752723.

8. Jose RJ, Manuel A. COVID-19 cytokine storm: the interplay between inflammation and coagulation. Lancet Respir Med 2020;8(6):e46e47. DOI: 10.1016/S2213-2600(20)30216-2Epub 2020 Apr 27. PMID: 32353251; PMCID: PMC7185942. 